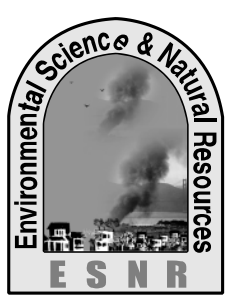

\section{Effects of Nitrogen Phosphorus Potassium and Sulphur on Growth Yield and Nutrient Content of Strawberry (Fragaria ananassa)}

\author{
C. A. Afroz ${ }^{1 *}$, M. A. H. Shimul ${ }^{2}$, M. Ikrum ${ }^{3}$, M. A. Siddiky ${ }^{4}$ and M. A. Razzaque ${ }^{1}$ \\ ${ }^{1}$ Department of Agricultural Chemistry \\ Sher-e-Bangla Agricultural University, Dhaka, Bangladesh \\ ${ }^{2,3}$ ACI Limited, Dhaka, Bangladesh \\ ${ }^{4}$ Horticulture Research Centre, BARI, Gazipur, Bangladesh \\ *Corresponding author: Chamon70@yahoo.com
}

\begin{abstract}
The experiment was conducted at Horticulture Research Centre, Gazipur, Bangladesh, to study the effects of N, P, K, and S on growth, yield and nutrient content of strawberry following Randomized Complete Block Design (RCBD) method. There were 4 levels of different nutrients and there was a positive impact of each fertilizer combinations on yield, yield parameters and nutrient contents of BARI Strawberry except control treatment. The highest values of plant height $(25.60 \mathrm{~cm})$; number of leaves (21.66), flowers (125.33), fruits (12.35),destroyed fruits (11), fruit weight $(215.10 \mathrm{~g})$ plant $^{-1}$ and fruit length $(4.16 \mathrm{~cm})$, fruit diameter $(3.41 \mathrm{~cm})$, individual fruit weight $(17.85 \mathrm{~g})$ and fruit yield $\left(11.50 \mathrm{t} \mathrm{ha}^{-1}\right)$ were found in treatment of $115,40,110$ and $25 \mathrm{~kg} \mathrm{ha}^{-1} \mathrm{NPKS}$, respectively. Among the fertilizers, the single effect of $\mathrm{N}\left(115 \mathrm{~kg} \mathrm{ha}^{-1}\right), \mathrm{P}\left(40 \mathrm{~kg} \mathrm{ha}^{-1}\right), \mathrm{K}\left(110 \mathrm{~kg} \mathrm{ha}^{-1}\right)$ and $\mathrm{S}\left(25 \mathrm{~kg} \mathrm{ha}^{-1}\right)$ gave maximum growth and yield of strawberry. The highest concentration of $\mathrm{N}, \mathrm{P}, \mathrm{K}$ and $\mathrm{S}$ were found in shoot and fruit of strawberry when $\mathrm{N}, \mathrm{P}, \mathrm{K}$ and $\mathrm{S}$ fertilizers were used 140,60,135 and $35 \mathrm{~kg} \mathrm{ha}^{-1}$, respectively.
\end{abstract}

Key words: BARI Strawberry, Growth, Nutrient content, Sulphur, Yield

\section{Introduction}

Strawberry (Fragaria ananassa) is a new fruit crop in Bangladesh which belongs to the family Rosaceae, is widely grown from the low- latitude tropics and subtropics to the colder high-latitude areas (Darnell et al., 2003). The plant comprises a shorten stem or crown from which arises leaves, runners, roots, auxiliary crowns and inflorescences (Darnell et al., 2003). Strawberries are native to most of the Northern hemisphere, including Europe and Britain. The garden (modern) strawberry is simply a hybrid of North and South American varieties. The cultivated strawberry is an octoploid $(2 \mathrm{n}=56)$ and though to be an autopolyploid (Albregts, et al., 1993). The leaves typically have three leaflets that means trifoliate. Strawberry fruit is non-climacteric and ripens rapidly (Wills et al., 1998). During ripening, fruit continue to increase in size, accumulate soluble solid content (SSC) and goes distinct changes on pigmentation and soften (Spayd and Morrus, 1981).

BARI Strawberry 1 is one of the high yielding varieties which is suitable for cultivation all over the country. The average yield is very low compared to that in other sub-tropical countries. Most of the soils and climatic conditions of Bangladesh are suitable for strawberry production but the crop did not show its potentially due to imbalance fertilization and poor agronomic practices. It is difficult to be specific about fertilizer recommendation because of variation in soil types, soil fertility and system of cultivation. Strawberry responds well to manuring and fertilizer. The application of high input technologies such as chemical fertilizers, pesticides, herbicides improved the production but there is growing concern over the adverse effects of the use of chemicals on soil productivity and environment quality (Prabhu et al., 2006). There is a scope for increasing yield of this crop with combination of $\mathrm{N}, \mathrm{P}$, $\mathrm{K}$ and $\mathrm{S}$ fertilizer under the agro-ecological condition in Bangladesh. Soil fertility and productivity changes overtime and this change towards negative direction because of intensive cropping with modern varieties, improper and imbalance use of fertilizer and manure (BARC, 2005). However, research works on nutrient management for strawberry is scanty in Bangladesh.

Hence the present study was, therefore, undertaken to evaluate the response to $\mathrm{N}, \mathrm{P}, \mathrm{K}$ and $\mathrm{S}$ and also to find out the optimum fertilizer package for maximizing yield of strawberry in Grey Terrace soil of Joydebpur Gazipur.

\section{Materials and Methods}

\section{Location of the experimental field}

The experiment was conducted at the Horticultural Research Farm, BARI, Joydebpur, Gazipur during robi season of 2011-2012.

\section{Experimental design}

The field experiment was set up in a Completely Randomized Block Design (RCBD) with three replications having 14 treatment combinations. Thus the total number of unit plots was 42 . The unit plot size was $2 \mathrm{~m} \times 1 \mathrm{~m}$. The plot to plot distance was $50 \mathrm{~cm} \times 50 \mathrm{~cm}$.

\section{Harvesting}

Ripen strawberry fruits were harvested from 1st March to 13th April, 2012. The necessary field data were recorded from eight selected plants at each treatment.

\section{Statistical analysis}

Data were statistically analyzed by analysis of variance (ANOVA) technique using the MSTAT-C Statistical Computer Package Programmee in accordance with the principles of Randomized Complete Block Design (RCBD). Ducan's Multiple Range Test (DMRT) was used to compare variations among the treatments.

\section{Results and Discussion}

This chapter contains results of the experiment and the follow-up discussion. For convenience, the whole 
chapter has been divided into two sections:

1. Agronomic characteristics

2. Chemical constituents.

Effects of different levels of nitrogen, phosphorus, potassium and Sulphur on growth and yield of strawberry

\section{Plant height}

The plant height was not significantly affected due to the combined effects of nitrogen, phosphorus, potassium and sulphur fertilizers application (Table 1). The result indicates that the height of strawberry plants was not significantly affected due to the single effects of nitrogen, phosphorus, potassium and sulphur fertilizer (Table 2a). But the height of plants also increased progressively with added $\mathrm{N}$ fertilizer up to $115 \mathrm{~kg} \mathrm{ha}^{-1}$. Further increase in fertilizer tended to depress plant height (Table 2a).

\section{Number of leaves}

Due to the combined effects of different levels of fertilizers significant difference was found on leaves number plant ${ }^{-1}$ (Table 1). The result (Table 2a) indicates that the leaf number of strawberry plants was significantly affected by different levels of $\mathrm{N}$ fertilizer.

\section{Number of flowers}

Different combinations of nitrogen, phosphorus, potassium and sulphur brought about significant $(\mathrm{p}<0.05)$ variation in regard to number of flowers plant

${ }^{1}$ (Table 1). The highest (125.33) number of flowers plant $^{-1}$ found from $T_{3}$ which is statistically similar with the treatment $\mathrm{T}_{4}$ The lowest number of flowers plant ${ }^{-1}$ (109.33) found from control treatments and was statistically similar to $\mathrm{T}_{8}$ and $\mathrm{T}_{5}$ treatments (Table 1 ).

Table 1. Effects of different fertilizer treatments on plant height, leaves plant ${ }^{-1}$ and flowers plant ${ }^{-1}$ of strawberry

\begin{tabular}{|c|c|c|c|}
\hline $\begin{array}{c}\text { Treatments } \\
\text { N-P-K-S kg ha }\end{array}$ & $\begin{array}{l}\text { Plant height } \\
\text { (cm) }\end{array}$ & Leaves plant $^{-1}$ (no.) & Flowers plant $^{-1}$ (no.) \\
\hline $\mathrm{T}_{1}(0-40-110-25)$ & 23.13 & $19.13 \mathrm{gh}$ & $114.33 \mathrm{e}-\mathrm{g}$ \\
\hline $\mathrm{T}_{2}(90-40-110-25)$ & 24.93 & $20.86 \mathrm{c}$ & $122.60 b$ \\
\hline $\mathrm{T}_{3}(115-40-110-25)$ & 25.60 & $21.66 \mathrm{a}$ & $125.33 a$ \\
\hline $\mathrm{T}_{4}(140-40-110-25)$ & 25.06 & $21.46 \mathrm{ab}$ & $124.00 \mathrm{ab}$ \\
\hline $\mathrm{T}_{5}(115-0-110-25)$ & 22.93 & $18.80 \mathrm{gh}$ & $113.66 \mathrm{gh}$ \\
\hline $\mathrm{T}_{6}(115-20-110-25)$ & 24.06 & $19.40 \mathrm{~d}-\mathrm{f}$ & $115.66 \mathrm{c}-\mathrm{e}$ \\
\hline $\mathrm{T}_{7}(115-60-110-25)$ & 24.13 & $20.33 c-e$ & $119.00 \mathrm{bc}$ \\
\hline $\mathrm{T}_{8}(115-40-0-25)$ & 22.80 & $18.40 \mathrm{gh}$ & $113.00 \mathrm{gh}$ \\
\hline $\mathrm{T}_{9}(115-40-85-25)$ & 23.86 & $19.33 \mathrm{~d}-\mathrm{e}$ & $115.06 \mathrm{c}-\mathrm{e}$ \\
\hline $\mathrm{T}_{10}(115-40-135-25)$ & 24.53 & $20.80 \mathrm{~cd}$ & $122.00 \mathrm{~b}$ \\
\hline $\mathrm{T}_{11}(115-40-110-0)$ & 23.33 & $19.26 \mathrm{e}-\mathrm{g}$ & $114.66 \mathrm{e}-\mathrm{g}$ \\
\hline $\mathrm{T}_{12}(115-40-110-15)$ & 24.46 & $20.66 \mathrm{~cd}$ & $120.00 \mathrm{bc}$ \\
\hline $\mathrm{T}_{13}(115-40-110-35)$ & 24.10 & $20.00 \mathrm{c}-\mathrm{e}$ & $117.00 \mathrm{bd}$ \\
\hline $\mathrm{T}_{14}(0-0-0-0)$ & 22.53 & $15.53 \mathrm{~h}$ & $109.33 \mathrm{~h}$ \\
\hline Significant level & NS & $*$ & $*$ \\
\hline $\mathrm{LSD}_{0.05}$ & - & 0.80 & 2.35 \\
\hline $\mathrm{CV}(\%)$ & 4.16 & 7.53 & 7.67 \\
\hline
\end{tabular}

In a column means having similar letter(s) are statistically identical and those having dissimilar letter(s) are differ significantly at 0.05 level of significance $\mathrm{NS} \longrightarrow$ Non significant, ${ }^{*} \longrightarrow 5 \%$ level of significance

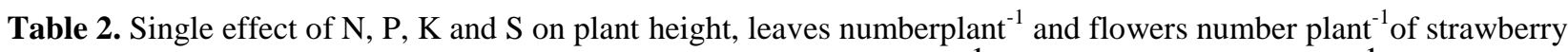
a) Effect of Nitrogen on plant height, leaves number plant ${ }^{-1}$ and flowers number plant ${ }^{-1}$

\begin{tabular}{|c|c|c|c|}
\hline $\begin{array}{l}\text { Nutrient levels } \\
\left(\mathrm{N} \mathrm{kg} \mathrm{ha}^{-1}\right)\end{array}$ & $\begin{array}{c}\text { Plant height } \\
(\mathrm{cm})\end{array}$ & Leaves plant $^{-1}$ (no.) & Flowers plant $^{-1}$ (no.) \\
\hline 0 & 23.13 & $19.13 \mathrm{~d}$ & $114.33 \mathrm{~d}$ \\
\hline 90 & 24.93 & $20.86 \mathrm{c}$ & $122.60 \mathrm{c}$ \\
\hline 115 & 25.60 & $21.66 \mathrm{a}$ & $125.33 \mathrm{a}$ \\
\hline 140 & 25.06 & $21.46 \mathrm{ab}$ & $124.0 \mathrm{ab}$ \\
\hline Significant level & NS & $*$ & $*$ \\
\hline $\mathrm{LSD}_{0.05}$ & - & 0.80 & 2.35 \\
\hline $\mathrm{CV}(\%)$ & 4.16 & 7.53 & 7.67 \\
\hline
\end{tabular}

In a column means having similar letter(s) are statistically identical and those having dissimilar letter(s) are differ significantly at 0.05 level of significance 
$\mathrm{NS} \rightarrow$ Non significant, $*$ $\rightarrow 5 \%$ level of significance

b) Effect of phosphorus on plant height, leaves number plant $^{-1}$ and flowers number plant ${ }^{-1}$

\begin{tabular}{|c|c|c|c|}
\hline $\begin{array}{l}\text { Nutrient levels } \\
\left(\mathbf{P ~ k g ~ h a}^{-1}\right)\end{array}$ & $\begin{array}{l}\text { Plant height } \\
\text { (cm) }\end{array}$ & Leaves plant $^{-1}$ (no.) & Flowers plant $^{-1}$ (no.) \\
\hline 0 & 22.93 & $18.80 \mathrm{~d}$ & $113.66 \mathrm{~d}$ \\
\hline 20 & 24.06 & $19.40 \mathrm{c}$ & $115.66 \mathrm{c}$ \\
\hline 40 & 25.60 & $21.66 \mathrm{a}$ & $125.33 \mathrm{a}$ \\
\hline 60 & 24.13 & $20.33 b$ & $119.00 \mathrm{~b}$ \\
\hline Significant level & NS & $*$ & $*$ \\
\hline $\operatorname{LSD}_{0.05}$ & - & 0.80 & 2.35 \\
\hline $\mathrm{CV}(\%)$ & 4.16 & 7.53 & 7.67 \\
\hline
\end{tabular}

In a column means having similar letter(s) are statistically identical and those having dissimilar letter(s) are differ significantly at 0.05 level of significance

$\mathrm{NS} \rightarrow$ Non significant, $\stackrel{*}{\longrightarrow} 5 \%$ level of significance

c) Effect of potassium on plant height, leaves number plant ${ }^{-1}$ and flowers number plant ${ }^{-1}$

\begin{tabular}{|c|c|c|c|}
\hline $\begin{array}{c}\text { Nutrient levels } \\
\left(\mathbf{K ~ k g ~ h a}^{-1}\right)\end{array}$ & $\begin{array}{c}\text { Plant height } \\
(\mathbf{c m})\end{array}$ & Leaves plant $^{\mathbf{- 1}}$ (no.) & Flowers plant $^{-\mathbf{1}}$ (no.) \\
\hline 0 & 22.80 & $18.40 \mathrm{~d}$ & $113.00 \mathrm{~d}$ \\
\hline 85 & 23.86 & $19.33 \mathrm{c}$ & $115.06 \mathrm{c}$ \\
\hline 110 & 25.60 & $21.66 \mathrm{a}$ & $125.33 \mathrm{a}$ \\
\hline 135 & 24.53 & $20.80 \mathrm{~b}$ & $122.00 \mathrm{~b}$ \\
\hline Significant level & NS & $*$ & $*$ \\
\hline LSD $_{0.05}$ & - & 0.80 & 2.35 \\
\hline $\mathrm{CV}(\%)$ & 4.16 & 7.53 & 7.67 \\
\hline
\end{tabular}

In a column means having similar letter(s) are statistically identical and those having dissimilar letter(s) are differ significantly at 0.05 level of significance

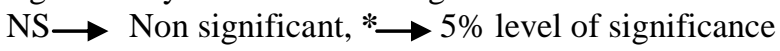

d) Effect of Sulphur on plant height, leaves number plant $^{-1}$ and flowers number plant ${ }^{-1}$

\begin{tabular}{|c|c|c|c|}
\hline $\begin{array}{c}\text { Nutrient levels } \\
\left(\mathbf{S ~ k g ~ h a}^{-\mathbf{1}}\right)\end{array}$ & $\begin{array}{c}\text { Plant height } \\
(\mathbf{c m})\end{array}$ & Leaves plant $^{-\mathbf{1}}$ (no.) & Flowers plant $^{-1}$ (no.) \\
\hline 0 & 23.33 & $19.26 \mathrm{c}$ & $114.66 \mathrm{~d}$ \\
\hline 15 & 24.46 & $20.66 \mathrm{~b}$ & $120.00 \mathrm{~b}$ \\
\hline 25 & 25.60 & $21.66 \mathrm{a}$ & $125.33 \mathrm{a}$ \\
\hline 35 & 24.10 & $20.00 \mathrm{bc}$ & $117.00 \mathrm{c}$ \\
\hline Significant level & NS & $*$ & 2.35 \\
\hline LSD $_{0.05}$ & - & 0.80 & 7.67 \\
\hline CV $(\%)$ & 4.16 & 7.53 & \\
\hline
\end{tabular}

In a column means having similar letter(s) are statistically identical and those having dissimilar letter(s) are differ significantly at 0.05 level of significance.

$\mathrm{NS} \rightarrow$ Non significant, $* \longrightarrow 5 \%$ level of significance

\section{Number of fruits}

Table 3. shows that the number of fruits plant $^{-1}$ significantly $(p<0.05)$ varied due to the combined effects of nitrogen, phosphorus, potassium and sulphur. This result indicates that the significant variation on number of fruits strawberry plant ${ }^{-1}$ under different levels of nitrogen (Table 4a).

\section{Fruit length and diameter}

There was a significant $(\mathrm{p}<0.05)$ variation on fruit length $(\mathrm{cm})$ due to the combined effects of nitrogen, phosphorus, potassium and sulphur fertilizers application (Table 3). The single effect of phosphorus showed significant $(\mathrm{p}<0.05)$ effect on fruit length of strawberry (Table $4 \mathrm{~b}$ ). Length of fruits increased with increasing rate of phosphorus up to $40 \mathrm{~kg} \mathrm{ha}^{-1}$ and then decreased fruit length with increasing rate of phosphorus (Table 4b). This result also indicates that the fruit length of strawberry significantly $(p<0.05)$ varied due to application of different levels of potassium (Table 4c). Fruit length increased with increasing rate of potassium upto $110 \mathrm{~kg} \mathrm{ha}^{-1}$, after that decreased fruit length (Table 4c). The present study indicates significant variation due to application of different levels of nitrogen on fruit diameter (Table 4a). Fruit diameter increased with increasing rate of nitrogen up to $115 \mathrm{~kg} \mathrm{ha}^{-1}$, after that increasing rate decreased fruit diameter (Table 4a). The result also indicates that the fruit diameter of strawberry significantly $(\mathrm{p}<0.05)$ varied due to application of different levels of potassium (Table 4c). 
Table 3. Effects of different fertilizer treatments on fruits no. plant ${ }^{-1}$, fruit length and fruit diameter of strawberry

\begin{tabular}{|c|c|c|c|}
\hline $\begin{array}{c}\text { Treatments } \\
\text { N-P-K-S Kg ha } \\
\end{array}$ & $\begin{array}{c}\text { Fruits plant }^{-1} \\
\text { (no.) }\end{array}$ & $\begin{array}{c}\text { Fruit length } \\
(\mathrm{cm})\end{array}$ & $\begin{array}{l}\text { Fruit diameter } \\
(\mathrm{cm})\end{array}$ \\
\hline $\mathrm{T}_{1}(0-40-110-25)$ & $8.15 \mathrm{~g}$ & $3.24 \mathrm{~cd}$ & $2.35 \mathrm{gh}$ \\
\hline $\mathrm{T}_{2}(90-40-110-25)$ & $11.55 b$ & $3.97 \mathrm{a}-\mathrm{c}$ & $3.25 \mathrm{ab}$ \\
\hline $\mathrm{T}_{3}(115-40-110-25)$ & $12.35 \mathrm{a}$ & $4.16 \mathrm{a}$ & $3.41 \mathrm{a}$ \\
\hline $\mathrm{T}_{4}(140-40-110-25)$ & $11.85 \mathrm{ab}$ & $4.08 \mathrm{ab}$ & $3.29 \mathrm{ab}$ \\
\hline $\mathrm{T}_{5}(115-0-110-25)$ & $8.45 f-h$ & $3.26 \mathrm{~cd}$ & $2.48 \mathrm{f}-\mathrm{h}$ \\
\hline $\mathrm{T}_{6}(115-20-110-25)$ & $9.95 \mathrm{c}-\mathrm{e}$ & $3.48 \mathrm{a}-\mathrm{d}$ & $2.88 b-f$ \\
\hline $\mathrm{T}_{7}(115-60-110-25)$ & $10.65 b-d$ & $3.65 a-d$ & $3.08 \mathrm{a}-\mathrm{d}$ \\
\hline $\mathrm{T}_{8}(115-40-0-25)$ & $9.05 \mathrm{e}-\mathrm{g}$ & $3.35 b-d$ & $2.55 \mathrm{e}-\mathrm{h}$ \\
\hline $\mathrm{T}_{9}(115-40-85-25)$ & $9.65 \mathrm{~d}-\mathrm{f}$ & $3.45 a-d$ & $2.75 \mathrm{c}-\mathrm{g}$ \\
\hline $\mathrm{T}_{10}(115-40-135-25)$ & $11.25 \mathrm{a}-\mathrm{c}$ & $3.90 a-d$ & $3.18 \mathrm{a}-\mathrm{c}$ \\
\hline $\mathrm{T}_{11}(115-40-110-0)$ & $9.35 \mathrm{~d}-\mathrm{g}$ & $3.41 \mathrm{a}-\mathrm{d}$ & $2.68 \mathrm{~d}-\mathrm{g}$ \\
\hline $\mathrm{T}_{12}(115-40-110-15)$ & $11.05 \mathrm{a}-\mathrm{c}$ & $3.78 \mathrm{a}-\mathrm{d}$ & $3.12 \mathrm{a}-\mathrm{d}$ \\
\hline $\mathrm{T}_{13}(115-40-110-35)$ & $10.35 c-e$ & $3.56 a-d$ & $2.95 \mathrm{a}-\mathrm{e}$ \\
\hline $\mathrm{T}_{14}(0-0-0-0)$ & $7.15 \mathrm{~h}$ & $3.15 \mathrm{~d}$ & $2.16 \mathrm{~h}$ \\
\hline Significant level & $*$ & $*$ & $*$ \\
\hline LSD $_{0.05}$ & 1.017 & 0.17 & 0.22 \\
\hline $\mathrm{CV}(\%)$ & 6.42 & 8.85 & 7.25 \\
\hline
\end{tabular}

In a column means having similar letter(s) are statistically identical and those having dissimilar letter(s) are differ significantly at 0.05 level of significance

$* \rightarrow 5 \%$ level of significance

Table 4. Single effect of N, P, K and S fertilizers on fruits no. plant ${ }^{-1}$, fruit length and fruit diameter of strawberry

a) Effects of nitrogen fruits no. plant ${ }^{-1}$, fruit length and fruit diameter

\begin{tabular}{|c|c|c|c|}
\hline $\begin{array}{c}\text { Nutrient levels } \\
\left.(\mathrm{N} \mathrm{kg} \mathrm{ha})^{-1}\right)\end{array}$ & Fruits plant $^{-1} \quad$ (no.) & $\begin{array}{c}\text { Fruit length } \\
(\mathrm{cm})\end{array}$ & $\begin{array}{c}\text { Fruit diameter } \\
(\mathrm{cm})\end{array}$ \\
\hline 0 & $8.15 \mathrm{c}$ & $3.24 \mathrm{c}$ & $2.35 \mathrm{c}$ \\
\hline 90 & $11.55 \mathrm{~b}$ & $3.97 b c$ & $3.25 b$ \\
\hline 115 & $12.35 \mathrm{a}$ & $4.16 \mathrm{a}$ & $3.41 \mathrm{a}$ \\
\hline 140 & $11.85 \mathrm{ab}$ & $4.08 \mathrm{ab}$ & $3.29 \mathrm{ab}$ \\
\hline Significant level & $*$ & $*$ & $*$ \\
\hline $\mathrm{LSD}_{0.05}$ & 1.017 & 0.17 & 0.22 \\
\hline $\mathrm{CV}(\%)$ & 6.42 & 8.85 & 7.25 \\
\hline
\end{tabular}

In a column means having similar letter(s) are statistically identical and those having dissimilar letter(s) are differ significantly at 0.05 level of significance* $\rightarrow 5 \%$ level of significance

b) Effects of phosphorus fruits no. plant $^{-1}$, fruit length and fruit diameter

\begin{tabular}{|c|c|c|c|}
\hline $\begin{array}{c}\text { Nutrient levels } \\
\left(\mathbf{P ~ k g ~ h a}^{-\mathbf{1}}\right)\end{array}$ & Fruits plant $^{\mathbf{1}} \quad$ (no.) $^{-}$ & $\begin{array}{c}\text { Fruit length } \\
(\mathbf{c m})\end{array}$ & $\begin{array}{c}\text { Fruit diameter } \\
(\mathbf{c m})\end{array}$ \\
\hline 0 & $8.45 \mathrm{~d}$ & $3.26 \mathrm{c}$ & $2.48 \mathrm{~d}$ \\
\hline 20 & $9.95 \mathrm{c}$ & $3.48 \mathrm{bc}$ & $2.88 \mathrm{c}$ \\
\hline 40 & $12.35 \mathrm{a}$ & $4.16 \mathrm{a}$ & $3.41 \mathrm{a}$ \\
\hline 60 & $10.65 \mathrm{~b}$ & $3.65 \mathrm{~b}$ & $3.08 \mathrm{~b}$ \\
\hline Significant level & $*$ & $*$ & $*$ \\
\hline LSD $_{0.05}$ & 1.017 & 0.17 & 0.22 \\
\hline CV $(\%)$ & 6.42 & 8.85 & 7.25 \\
\hline
\end{tabular}

In a column means having similar letter(s) are statistically identical and those having dissimilar letter(s) are differ significantly at 0.05 level of significance* $\rightarrow 5 \%$ level of significance 
c) Effects of potassium fruits no. plant $^{-1}$, fruit length and fruit diameter

\begin{tabular}{|c|c|c|c|}
\hline $\begin{array}{c}\text { Nutrient levels } \\
\left(\mathbf{K ~ k g ~ h a}^{-\mathbf{1}}\right)\end{array}$ & Fruits plant $^{\mathbf{- 1}}$ (no.) & $\begin{array}{c}\text { Fruit length } \\
(\mathbf{c m})\end{array}$ & $\begin{array}{c}\text { Fruit diameter } \\
(\mathbf{c m})\end{array}$ \\
\hline 0 & $9.05 \mathrm{~d}$ & $3.35 \mathrm{~cd}$ & $2.55 \mathrm{~d}$ \\
\hline 85 & $9.65 \mathrm{c}$ & $3.45 \mathrm{c}$ & $2.75 \mathrm{c}$ \\
\hline 110 & $12.35 \mathrm{a}$ & $4.16 \mathrm{a}$ & $3.41 \mathrm{a}$ \\
\hline 135 & $11.25 \mathrm{~b}$ & $3.90 \mathrm{~b}$ & $3.18 \mathrm{~b}$ \\
\hline Significant level & $*$ & $*$ & $*$ \\
\hline LSD $_{0.05}$ & 1.017 & 0.17 & 0.22 \\
\hline CV (\%) & 6.42 & 8.85 & 7.25 \\
\hline
\end{tabular}

In a column means having similar letter(s) are statistically identical and those having dissimilar letter(s) are differ significantly at 0.05 level of significance* $\rightarrow 5 \%$ level of significance

d) Effects of sulphur fruits no. plant ${ }^{-1}$, fruit length and fruit diameter

\begin{tabular}{|c|c|c|c|}
\hline $\begin{array}{l}\text { Nutrient levels } \\
\left(\mathrm{S} \mathrm{kg} \mathrm{ha}^{-1}\right)\end{array}$ & Fruits plant $^{-1} \quad$ (no.) & $\begin{array}{l}\text { Fruit length } \\
(\mathrm{cm})\end{array}$ & $\begin{array}{c}\text { Fruit diameter } \\
(\mathbf{c m})\end{array}$ \\
\hline 0 & $9.35 \mathrm{~d}$ & $3.41 \mathrm{~d}$ & $2.68 \mathrm{~d}$ \\
\hline 15 & $11.05 \mathrm{~b}$ & $3.78 \mathrm{~b}$ & $3.12 b$ \\
\hline 25 & $12.35 \mathrm{a}$ & $4.16 \mathrm{a}$ & $3.41 \mathrm{a}$ \\
\hline 35 & $10.35 \mathrm{c}$ & $3.56 \mathrm{c}$ & $2.95 \mathrm{c}$ \\
\hline Significant level & $*$ & $*$ & $*$ \\
\hline $\mathrm{LSD}_{0.05}$ & 1.017 & 0.17 & 0.22 \\
\hline $\mathrm{CV}(\%)$ & 6.42 & 8.85 & 7.25 \\
\hline
\end{tabular}

In a column means having similar letter(s) are statistically identical and those having dissimilar letter(s) are differ significantly at 0.05 level of significance*

\section{Number of damaged fruits}

Number of damaged fruits plant ${ }^{-1}$ of BARI Strawberry 1 varied significantly $(p<0.05)$ due to the combined effects of nitrogen, phosphorus, potassium and sulphur (Table 5). Data indicates that damaged fruit number plant $^{-1}$ varied significantly $(\mathrm{p}<0.05)$ by the application of different levels of phosphorus (Table 6b). The effect of different levels of potassium showed significant variation on the number of damaged fruitsplant ${ }^{-1}$ (Table 6c).

Table 5. Effects of different fertilizer treatments on damaged fruits plant ${ }^{-1}$, individual fruit weight, fruit weight plant ${ }^{1}$ and yield of strawberry

\begin{tabular}{|c|c|c|c|c|}
\hline $\begin{array}{c}\text { Treatments } \\
\text { N-P-K-S Kg ha }\end{array}$ & $\begin{array}{l}\text { Damaged fruits } \\
\text { plant }^{-1} \text { (no.) }\end{array}$ & $\begin{array}{l}\text { Individual fruit } \\
\text { wt. (g) }\end{array}$ & Fruit wt. plant $^{-1}(\mathrm{~g})$ & $\begin{array}{c}\text { Yield } \\
\left(\mathrm{t} \mathrm{ha}^{-1}\right)\end{array}$ \\
\hline $\mathrm{T}_{1}(0-40-110-25)$ & $9.32 \mathrm{~d}-\mathrm{e}$ & $12.80 \mathrm{gh}$ & $142.65 \mathrm{gh}$ & $8.95 \mathrm{ef}$ \\
\hline $\mathrm{T}_{2}(90-40-110-25)$ & $10.33 a-c$ & $16.80 \mathrm{a}-\mathrm{c}$ & $199.65 a-c$ & $11.10 \mathrm{ab}$ \\
\hline $\mathrm{T}_{3}(115-40-110-25)$ & $11.00 \mathrm{a}$ & $17.85 \mathrm{a}$ & $215.10 \mathrm{a}$ & $11.50 \mathrm{a}$ \\
\hline $\mathrm{T}_{4}(140-40-110-25)$ & $10.66 \mathrm{ab}$ & $17.40 \mathrm{ab}$ & $205.95 \mathrm{ab}$ & $11.32 \mathrm{ab}$ \\
\hline $\mathrm{T}_{5}(115-0-110-25)$ & $9.00 \mathrm{e}-\mathrm{g}$ & $13.20 f-h$ & $145.45 \mathrm{f}-\mathrm{h}$ & $9.15 \mathrm{~d}-\mathrm{f}$ \\
\hline $\mathrm{T}_{6}(115-20-110-25)$ & $9.63 b-d$ & $15.20 \mathrm{~b}-\mathrm{f}$ & $165.35 \mathrm{c}-\mathrm{g}$ & $10.15 a-e$ \\
\hline $\mathrm{T}_{7}(115-60-110-25)$ & $9.66 b-d$ & $15.90 \mathrm{a}-\mathrm{e}$ & $180.95 a-f$ & $10.48 a-e$ \\
\hline $\mathrm{T}_{8}(115-40-0-25)$ & $8.00 \mathrm{~h}$ & $13.75 \mathrm{e}-\mathrm{g}$ & $152.45 f-h$ & $9.38 \mathrm{~d}-\mathrm{f}$ \\
\hline $\mathrm{T}_{9}(115-40-85-25)$ & $9.60 b-d$ & $14.85 \mathrm{c}-\mathrm{g}$ & $160.35 d-h$ & $9.90 b-e$ \\
\hline $\mathrm{T}_{10}(115-40-135-25)$ & $10.30 \mathrm{a}-\mathrm{c}$ & $16.40 \mathrm{a}-\mathrm{d}$ & 195.80a-d & $10.95 a-c$ \\
\hline $\mathrm{T}_{11}(115-40-110-0)$ & $9.33 \mathrm{~d}-\mathrm{f}$ & $14.35 \mathrm{~d}-\mathrm{g}$ & $155.65 \mathrm{e}-\mathrm{h}$ & $9.62 c-f$ \\
\hline $\mathrm{T}_{12}(115-40-110-15)$ & $10.00 \mathrm{~b}-\mathrm{d}$ & $16.10 \mathrm{a}-\mathrm{d}$ & $185.75 a-e$ & $10.68 \mathrm{a}-\mathrm{d}$ \\
\hline $\mathrm{T}_{13}(115-40-110-35)$ & $9.61 b-d$ & $15.55 a-e$ & $175.30 \mathrm{~b}-\mathrm{g}$ & $10.70 a-e$ \\
\hline $\mathrm{T}_{14}(0-0-0-0)$ & $6.00 \mathrm{i}$ & $11.10 \mathrm{~h}$ & $125.35 \mathrm{~h}$ & $8.20 \mathrm{f}$ \\
\hline Significant level & $*$ & $*$ & $*$ & $*$ \\
\hline $\mathrm{LSD}_{0.05}$ & 1.01 & 1.69 & 25.24 & 1.012 \\
\hline $\mathrm{CV}(\%)$ & 8.44 & 7.9 & 8.45 & 9.95 \\
\hline
\end{tabular}

In a column means having similar letter(s) are statistically identical and those having dissimilar letter(s) are differ significantly at 0.05 level of significance 
$* \quad \rightarrow 5 \%$ level of significance

\section{Individual fruit weight}

Table 4.5showed a significant $(\mathrm{p}<0.05)$ variation in individual fruit weight $(\mathrm{g})$ by the combined application of nitrogen, phosphorus, potassium and Sulphur fertilizers. Table $4.6 \mathrm{c}$ showed a significant $(\mathrm{p}<0.05)$ variation in individual fruit weight $(\mathrm{g})$ by the application of different levels of potassium. Individual fruit weight linearly increased with increasing level of $\mathrm{K}$ doses up to $110 \mathrm{~kg} \mathrm{ha}^{-1}$ and after that gave lower individual fruit weight (Table 6c).

\section{Fruit weight plant ${ }^{-1}$}

Fruit weight $\operatorname{plant}^{-1}(g)$ was significantly $\quad(\mathrm{p}<0.05)$ affected due to the combined application of nitrogen, phosphorus, potassium and sulphur fertilizers (Table 5).
The single effect of different levels of nitro N,P,K,S showed significant variation on the of fruit weight plant ${ }^{1}$ (Table 6a).

\section{Fruit yield}

Fruit yield per hectare was significantly $(p<0.05)$ influenced due to the combined effects of nitrogen, phosphorus, potassium and sulphur fertilizers application (Table 5). Fruit yield was significantly $(p<0.05)$ affected by the application of different levels of nitrogen fertilizer (Table 7a). The single effect of different levels of phosphorus showed significant variation on the fruit yield (Table $7 b$ ). Fruit yield was significantly $(\mathrm{p}<0.05)$ affected by application of different levels of potassium fertilizer (Table 7c).

Table 6. Single effect of N, P, K and S on damaged fruits plant ${ }^{-1}$, individual fruit weight, fruit weight plant ${ }^{-1}$ of strawberry

a) Effects of nitrogenon damaged fruits plant ${ }^{-1}$, individual fruit weight and fruit weight plant ${ }^{-1}$

\begin{tabular}{|c|c|c|c|}
\hline $\begin{array}{c}\text { Nutrient levels } \\
\left(\mathrm{N} \text { kg ha }{ }^{-1}\right)\end{array}$ & $\begin{array}{c}\text { Damaged fruits } \\
\text { plant }^{-1} \text { (no.) }\end{array}$ & $\begin{array}{c}\text { Fruit wt. plant }^{-1} \\
\text { (g) }\end{array}$ & $\begin{array}{c}\text { Individual fruit wt. } \\
\text { (g) }\end{array}$ \\
\hline 0 & $9.32 \mathrm{c}$ & $142.65 \mathrm{c}$ & $12.80 \mathrm{c}$ \\
\hline 90 & $10.33 b c$ & $199.65 b$ & $16.80 \mathrm{~b}$ \\
\hline 115 & $11.00 \mathrm{a}$ & $215.10 \mathrm{a}$ & $17.85 \mathrm{a}$ \\
\hline 140 & $10.66 \mathrm{~b}$ & $205.95 \mathrm{ab}$ & $17.40 \mathrm{ab}$ \\
\hline Significant level & $*$ & $*$ & $*$ \\
\hline $\mathrm{LSD}_{0.05}$ & 1.01 & 25.24 & 1.69 \\
\hline $\mathrm{CV}(\%)$ & 8.44 & 8.45 & 7.9 \\
\hline
\end{tabular}

In a column means having similar letter(s) are statistically identical and those having dissimilar letter(s) are differ significantly at 0.05 level of significance* $\rightarrow 5 \%$ level of significance

b) Effects of phosphoruson damaged fruits plant ${ }^{-1}$, individual fruit weight and fruit weight plant $^{-1}$

\begin{tabular}{|c|c|c|c|}
\hline $\begin{array}{c}\text { Nutrient levels (P kg } \\
\text { ha }^{-1} \text { ) }\end{array}$ & $\begin{array}{c}\text { Damaged fruits plant } \\
\text { (no.) }\end{array}$ & $\begin{array}{c}\text { Fruit wt. plant } \\
\text { (g) }\end{array}$ & $\begin{array}{c}\text { Individual fruit wt. } \\
\text { (g) }\end{array}$ \\
\hline 0 & $9.00 \mathrm{c}$ & $145.45 \mathrm{~d}$ & $13.20 \mathrm{~d}$ \\
\hline 20 & $9.63 \mathrm{bc}$ & $165.35 \mathrm{c}$ & $15.20 \mathrm{c}$ \\
\hline 40 & $11.00 \mathrm{a}$ & $215.10 \mathrm{a}$ & $17.85 \mathrm{a}$ \\
\hline 60 & $9.66 \mathrm{~b}$ & $180.95 b$ & $15.90 \mathrm{~b}$ \\
\hline Significant level & $*$ & $*$ & $*$ \\
\hline LSD $_{0.05}$ & 1.01 & 25.24 & 1.69 \\
\hline $\mathrm{CV}(\%)$ & 8.44 & 8.45 & 7.9 \\
\hline
\end{tabular}

In a column means having similar letter(s) are statistically identical and those having dissimilar letter(s) are differ significantly at 0.05 level of significance* $\rightarrow 5 \%$ level of significance

c) Effect of potassium on damaged fruits plant ${ }^{-1}$, individual fruit weight and fruit weight plant ${ }^{-1}$

\begin{tabular}{|c|c|c|c|}
\hline $\begin{array}{l}\text { Nutrient levels } \\
\left(\mathrm{K} \mathrm{kg} \mathrm{ha}^{-1}\right)\end{array}$ & $\begin{array}{c}\text { Damaged fruits plant } \\
\text { (no.) }\end{array}$ & $\begin{array}{c}\text { Fruit wt. plant } \\
\text { (g) }\end{array}$ & $\begin{array}{c}\text { Individual fruit wt. } \\
\text { (g) }\end{array}$ \\
\hline 0 & $8.00 \mathrm{~d}$ & $152.45 \mathrm{~d}$ & $13.75 \mathrm{~d}$ \\
\hline 85 & $9.60 \mathrm{c}$ & $160.35 \mathrm{c}$ & $14.85 \mathrm{c}$ \\
\hline 110 & $11.00 \mathrm{a}$ & $215.10 \mathrm{a}$ & $17.85 \mathrm{a}$ \\
\hline 135 & $10.30 \mathrm{~b}$ & $195.80 \mathrm{~b}$ & $16.40 \mathrm{~b}$ \\
\hline Significant level & $*$ & $*$ & $*$ \\
\hline $\mathrm{LSD}_{0.05}$ & 1.01 & 25.24 & 1.69 \\
\hline $\mathrm{CV}(\%)$ & 8.44 & 8.45 & 7.9 \\
\hline
\end{tabular}

In a column means having similar letter(s) are statistically identical and those having dissimilar letter(s) are differ significantly at 0.05 level of significance* $\rightarrow 5 \%$ level of significance 
d) Effect of sulphur on damaged fruits plant ${ }^{-1}$, individual fruit weight and fruit weight plant ${ }^{-1}$

\begin{tabular}{|c|c|c|c|}
\hline $\begin{array}{l}\text { Nutrient levels } \\
\left(\mathrm{S} \mathrm{kg} \mathrm{ha}^{-1}\right)\end{array}$ & $\begin{array}{c}\text { Damaged fruits plant }^{-1} \\
\text { (no.) }\end{array}$ & $\begin{array}{l}\text { Fruit wt. plant }^{-1} \\
\text { (g) }\end{array}$ & $\begin{array}{l}\text { Individual fruit wt. } \\
\text { (g) }\end{array}$ \\
\hline 0 & $9.33 \mathrm{c}$ & $155.65 \mathrm{~d}$ & $14.35 \mathrm{~d}$ \\
\hline 15 & $10.00 \mathrm{ab}$ & $185.75 \mathrm{~b}$ & $16.10 \mathrm{~b}$ \\
\hline 25 & $11.00 \mathrm{a}$ & $215.10 \mathrm{a}$ & $17.85 \mathrm{a}$ \\
\hline 35 & $9.61 \mathrm{~b}$ & $175.30 \mathrm{c}$ & $15.55 \mathrm{c}$ \\
\hline Significant level & $*$ & $*$ & $*$ \\
\hline LSD $_{0.05}$ & 1.01 & 25.24 & 1.69 \\
\hline $\mathrm{CV}(\%)$ & 8.44 & 8.45 & 7.9 \\
\hline
\end{tabular}

In a column means having similar letter(s) are statistically identical and those having dissimilar letter(s) are differ significantly at 0.05 level of significance* $\rightarrow 5 \%$ level of significance

Table 7. Single effect of N, P, K and S on yield of strawberry

a) Effect of nitrogen on yield of strawberry

\begin{tabular}{|c|c|c|}
\hline $\begin{array}{c}\text { Nutrient levels } \\
\left(\mathbf{N ~ k g ~ h a}^{-1}\right)\end{array}$ & $\begin{array}{c}\text { Fruit yield } \\
\left(\mathbf{t ~ h a}^{-\mathbf{1}}\right)\end{array}$ & \% Yield increase over control \\
\hline 0 & $8.95 \mathrm{c}$ & - \\
\hline 90 & $11.10 \mathrm{~b}$ & 24 \\
\hline 115 & $11.50 \mathrm{a}$ & 28 \\
\hline 140 & $11.32 \mathrm{ab}$ & - \\
\hline Significant level & $*$ & - \\
\hline LSD $_{0.05}$ & 1.012 & - \\
\hline $\mathrm{CV}$ & 9.95 & 26 \\
\hline
\end{tabular}

In a column means having similar letter(s) are statistically identical and those having dissimilar letter(s) are differ significantly at 0.05 level of significance* $\rightarrow 5 \%$ level of significance

b) Effect of phosphorus on yield of strawberry

\begin{tabular}{|c|c|c|}
\hline $\begin{array}{c}\text { Nutrient levels } \\
\left(\mathbf{P ~ k g ~ h a}^{\mathbf{- 1}}\right)\end{array}$ & $\begin{array}{c}\text { Fruit yield } \\
\left(\mathbf{t ~ h a}^{-\mathbf{1}}\right)\end{array}$ & \% Yield increase over control \\
\hline 0 & $9.15 \mathrm{~d}$ & - \\
\hline 20 & $10.15 \mathrm{c}$ & 11 \\
\hline 40 & $11.50 \mathrm{a}$ & 26 \\
\hline 60 & $10.48 \mathrm{~b}$ & 15 \\
\hline Significant level & $*$ & - \\
\hline LSD $_{0.05}$ & 1.012 & - \\
\hline CV\% & 9.95 & - \\
\hline
\end{tabular}

In a column means having similar letter(s) are statistically identical and those having dissimilar letter(s) are differ significantly at 0.05 level of significance* $\rightarrow 5 \%$ level of significance

c) Effect of potassium on yield of strawberry

\begin{tabular}{|c|c|c|}
\hline $\begin{array}{c}\text { Nutrient levels } \\
\left(\mathrm{K} \mathrm{kg} \mathrm{ha}^{-\mathbf{1}}\right)\end{array}$ & $\begin{array}{c}\text { Fruit yield } \\
\left(\mathbf{t ~ h a}^{-\mathbf{1}}\right)\end{array}$ & \% Yield increase over control \\
\hline 0 & $9.38 \mathrm{~d}$ & - \\
\hline 85 & $9.90 \mathrm{c}$ & 5 \\
\hline 110 & $11.50 \mathrm{a}$ & 23 \\
\hline 135 & $10.95 \mathrm{~b}$ & 17 \\
\hline Significant level & $*$ & - \\
\hline LSD $_{0.05}$ & 1.012 & - \\
\hline $\mathrm{CV} \%$ & 9.95 & - \\
\hline
\end{tabular}

In a column means having similar letter(s) are statistically identical and those having dissimilar letter(s) are differ significantly at 0.05 level of significance

$* \rightarrow 5 \%$ level of significance 
d) Effect of sulphur on yield of strawberry

\begin{tabular}{|c|c|c|}
\hline $\begin{array}{c}\text { Nutrient levels } \\
\left(\mathbf{S ~ k g ~ h a}^{-\mathbf{1}}\right)\end{array}$ & $\begin{array}{c}\text { Fruit yield } \\
\left(\mathbf{t ~ h a}^{\mathbf{- 1}}\right)\end{array}$ & \% Yield increase over control \\
\hline 0 & $9.62 \mathrm{c}$ & - \\
\hline 15 & $10.70 \mathrm{~b}$ & 10 \\
\hline 25 & $11.50 \mathrm{a}$ & 20 \\
\hline 35 & $10.68 \mathrm{c}$ & 11 \\
\hline Significant level & $*$ & - \\
\hline $\mathrm{LSD}_{0.05}$ & 1.012 & - \\
\hline $\mathrm{CV} \%$ & 9.95 & - \\
\hline
\end{tabular}

In a column means having similar letter(s) are statistically identical and those having dissimilar letter(s) are differ significantly at 0.05 level of significance

$* \rightarrow 5 \%$ level of significance

\section{$N, P, K, S$ content in shoot and fruit}

The effect of nitrogen on nitrogen content in shoot of strawberry was statistically significant due to the different combined doses of $\mathrm{N}, \mathrm{P}, \mathrm{K}$ and $\mathrm{S}$ fertilizers (Table 8 and Table 9). Data shows that nitrogen content in shoot increased with increasing level of nitrogen application. In case of strawberry fruit the effect of combined doses of $\mathrm{N}, \mathrm{P}, \mathrm{K}$ and $\mathrm{S}$ fertilizers varied significantly on $\mathrm{N}$ content (Table 9). It was observed that the $\mathrm{N}$ content gradually increased with treatment
$\mathrm{T}_{4}$ the content of nitrogen was the highest $(2.06 \%)$ and statistically similar to treatment $\mathrm{T}_{3}$. In the fruit of strawberry, the $\mathrm{S}$ content did not significantly differ by the application of different levels of $\mathrm{N}, \mathrm{P}, \mathrm{K}$ and $\mathrm{S}$ fertilizers (Table 9$)$. The highest $\mathrm{S}$ content $(0.35 \%)$ was found in $\mathrm{T}_{13}$ treatment. The lowest $\mathrm{S}$ content $(0.12 \%)$ was found in control. The result is in agreement with the findings of Chandal et al. (2003) that increasing S levels significantly increased $\mathrm{S}$ content up to $45 \mathrm{~kg} \mathrm{~S}$ $\mathrm{ha}^{-1}$.

Table 8. Effects of N, P, K and S application on nutrient content of strawberry shoot

\begin{tabular}{|c|c|c|c|c|}
\hline \multirow{2}{*}{$\begin{array}{c}\begin{array}{c}\text { Treatments } \\
\left(\mathbf{k g ~ h a}^{-1}\right)\end{array} \\
\mathbf{N}-\mathbf{P}-\mathbf{K}-\mathbf{S}\end{array}$} & \multicolumn{4}{|c|}{ Nutrient content $(\%)$ in root } \\
\hline & $\mathbf{N}$ & $\mathbf{P}$ & $\mathbf{K}$ & $\mathbf{S}$ \\
\hline $\mathrm{T}_{1}(0-40-110-25)$ & $0.58 \mathrm{e}$ & $0.44 \mathrm{e}$ & $1.67 \mathrm{~g}$ & 0.20 \\
\hline $\mathrm{T}_{2}(90-40-110-25)$ & $1.25 \mathrm{~cd}$ & $0.59 \mathrm{~cd}$ & $2.58 \mathrm{~d}$ & 0.22 \\
\hline $\mathrm{T}_{3}(115-40-110-25)$ & $1.62 \mathrm{a}$ & $0.80 \mathrm{a}$ & $2.99 \mathrm{a}$ & 0.30 \\
\hline $\mathrm{T}_{4}(140-40-110-25)$ & $1.68 \mathrm{a}$ & $0.70 \mathrm{~b}$ & $2.72 \mathrm{c}$ & 0.27 \\
\hline $\mathrm{T}_{5}(115-0-110-25)$ & $1.32 \mathrm{bc}$ & $0.38 \mathrm{f}$ & $1.89 \mathrm{e}$ & 0.15 \\
\hline $\mathrm{T}_{6}(115-20-110-25)$ & $1.39 \mathrm{~b}$ & $0.65 \mathrm{c}$ & $2.70 \mathrm{c}$ & 0.24 \\
\hline $\mathrm{T}_{7}(115-60-110-25)$ & $1.47 \mathrm{~b}$ & $0.84 \mathrm{a}$ & $2.89 \mathrm{~b}$ & 0.28 \\
\hline $\mathrm{T}_{8}(115-40-0-25)$ & $1.28 \mathrm{~cd}$ & $0.46 \mathrm{e}$ & $1.28 \mathrm{~h}$ & 0.21 \\
\hline $\mathrm{T}_{9}(115-40-85-25)$ & $1.35 \mathrm{~b}$ & $0.63 \mathrm{c}$ & $1.88 \mathrm{e}$ & 0.25 \\
\hline $\mathrm{T}_{10}(115-40-135-25)$ & $1.44 \mathrm{~b}$ & $0.74 \mathrm{~b}$ & $3.07 \mathrm{a}$ & 0.26 \\
\hline $\mathrm{T}_{11}(115-40-110-0)$ & $1.27 \mathrm{~cd}$ & $0.47 \mathrm{e}$ & $1.51 \mathrm{f}$ & 0.15 \\
\hline $\mathrm{T}_{12}(115-40-110-15)$ & $1.37 \mathrm{~b}$ & $0.62 \mathrm{c}$ & $2.52 \mathrm{de}$ & 0.18 \\
\hline $\mathrm{T}_{13}(115-40-110-35)$ & $1.41 \mathrm{~b}$ & $0.72 \mathrm{~b}$ & $2.82 \mathrm{~b}$ & 0.31 \\
\hline $\mathrm{T}_{14}(0-0-0-0)$ & $0.48 \mathrm{e}$ & $0.05 \mathrm{~g}$ & $0.90 \mathrm{i}$ & 0.11 \\
\hline Significant level & $*$ & $*$ & $*$ & NS \\
\hline LSD $_{0.05}$ & 0.14 & 0.06 & 0.08 & - \\
\hline CV $(\%)$ & 2.21 & 3.16 & 2.82 & 5.11 \\
\hline
\end{tabular}

In a column means having similar letter(s) are statistically identical and those having dissimilar letter(s) are differ significantly at 0.05 level of significance

NS $\rightarrow$ Non significant, $* \longrightarrow 5 \%$ level of significant 
Table 9. Effects of N, P, K and S application on nutrient content of strawberry fruit

\begin{tabular}{|c|c|c|c|c|}
\hline \multirow{2}{*}{$\begin{array}{c}\text { Treatments }\left(\mathrm{kg} \mathrm{ha}^{-1}\right) \\
\mathbf{N}-\mathbf{P}-\mathbf{K}-\mathbf{S}\end{array}$} & \multicolumn{4}{|c|}{ Nutrient content $(\%)$ in fruit } \\
\hline & $\mathbf{N}$ & $\mathbf{P}$ & $\mathbf{K}$ & $\mathbf{S}$ \\
\hline $\mathrm{T}_{1}(0-40-110-25)$ & $0.42 \mathrm{e}$ & $0.63 \mathrm{~cd}$ & $1.29 \mathrm{~g}$ & 0.16 \\
\hline $\mathrm{T}_{2}(90-40-110-25)$ & $1.12 \mathrm{~d}$ & $0.66 \mathrm{c}$ & $1.38 \mathrm{e}$ & 0.21 \\
\hline $\mathrm{T}_{3}(115-40-110-25)$ & $1.98 \mathrm{a}$ & $0.82 \mathrm{a}$ & $1.70 \mathrm{a}$ & 0.32 \\
\hline $\mathrm{T}_{4}(140-40-110-25)$ & $2.06 \mathrm{a}$ & $0.71 \mathrm{bc}$ & $1.65 \mathrm{~b}$ & 0.29 \\
\hline $\mathrm{T}_{5}(115-0-110-25)$ & $0.96 \mathrm{de}$ & $0.46 \mathrm{~d}$ & $1.36 \mathrm{f}$ & 0.17 \\
\hline $\mathrm{T}_{6}(115-20-110-25)$ & $1.65 \mathrm{~b}$ & $0.74 \mathrm{bc}$ & $1.41 \mathrm{de}$ & 0.23 \\
\hline $\mathrm{T}_{7}(115-60-110-25)$ & $1.57 \mathrm{bc}$ & $0.88 \mathrm{a}$ & $1.58 \mathrm{c}$ & 0.26 \\
\hline $\mathrm{T}_{8}(115-40-0-25)$ & $0.78 \mathrm{de}$ & $0.59 \mathrm{~cd}$ & $1.18 \mathrm{~h}$ & 0.16 \\
\hline $\mathrm{T}_{9}(115-40-85-25)$ & $1.42 \mathrm{~cd}$ & $0.62 \mathrm{~cd}$ & $1.45 \mathrm{~d}$ & 0.19 \\
\hline $\mathrm{T}_{10}(115-40-135-25)$ & $1.58 \mathrm{bc}$ & $0.76 \mathrm{~b}$ & $1.77 \mathrm{a}$ & 0.24 \\
\hline $\mathrm{T}_{11}(115-40-110-0)$ & $0.84 \mathrm{de}$ & $0.61 \mathrm{~cd}$ & $1.34 \mathrm{f}$ & 0.14 \\
\hline $\mathrm{T}_{12}(115-40-110-15)$ & $1.45 \mathrm{~cd}$ & $0.68 \mathrm{c}$ & $1.48 \mathrm{~d}$ & 0.24 \\
\hline $\mathrm{T}_{13}(115-40-110-35)$ & $1.65 \mathrm{~b}$ & $0.78 \mathrm{~b}$ & $1.56 \mathrm{c}$ & 0.35 \\
\hline $\mathrm{T}_{14}(0-0-0-0)$ & $0.28 f$ & $0.38 \mathrm{e}$ & $1.09 \mathrm{i}$ & 0.12 \\
\hline Significant level & $*$ & $*$ & $*$ & NS \\
\hline LSD $_{0.05}$ & 0.22 & 0.04 & 0.04 & - \\
\hline $\mathrm{CV}(\%)$ & 2.34 & 3.12 & 3.43 & 5.51 \\
\hline
\end{tabular}

In a column means having similar letter(s) are statistically identical and those having dissimilar letter(s) are differ significantly at 0.05 level of significance

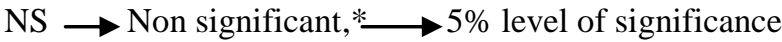

\section{Regression analysis}

Regression analysis of strawberry yield was done to fit the quadratic functions for estimating the optimum levels of each nutrient over the different levels of NPKS $\mathrm{ha}^{-1}$ (Fig 4.1 and Fig 4.2). From the regression equation the agronomic and economically optimum levels of NPKS were estimated as $125,46,117$ and $23 \mathrm{~kg} \mathrm{ha}^{-1}$, and
$120,45,115$ and $20 \mathrm{~kg}$ NPKS ha ${ }^{-1}$ respectively (Table 10).

The present result indicates that judicious nutrient management in strawberry can ensure high persecution and profit. Application of 120,45,115 and $20 \mathrm{~kg}$ NPKS $\mathrm{ha}^{-1}$ would be the beneficial and economically optimum for strawberry production in Grey Terrace Soil of Joydebpur under AEZ 28.

Table 10. Response function of strawberry yield to N, P, K and S fertilizers

\begin{tabular}{|c|l|l|c|c|}
\hline \multirow{2}{*}{ Nutrients } & Regression equation & $\mathbf{R}^{\mathbf{2}}$ & \multicolumn{2}{c|}{ Optimum rates of nutrient $\left(\mathrm{kg} \mathrm{ha}^{\mathbf{- 1}}\right)$} \\
\cline { 3 - 5 } & & & Agronomic & Economic \\
\hline $\mathrm{N}$ & $\mathrm{Y}=8.0943+0.025 \mathrm{x}-0.0001 \mathrm{x}^{2}$ & 0.993 & 125 & 120 \\
\hline $\mathrm{P}$ & $\mathrm{Y}=9.014+0.092 \mathrm{x}-0.001 \mathrm{x}^{2}$ & 0.948 & 46 & 45 \\
\hline $\mathrm{K}$ & $\mathrm{Y}=9.363+0.0234 \mathrm{x}-0.0001 \mathrm{x}^{2}$ & 0.937 & 117 & 115 \\
\hline $\mathrm{S}$ & $\mathrm{Y}=9.557+0.091 \mathrm{x}-0.002 \mathrm{x}^{2}$ & 0.957 & 23 & 20 \\
\hline
\end{tabular}

\section{Conclusions}

There was no significant difference on plant height of strawberry plant on the application different combinations of fertilizers. However, the tallest plant $(25.60 \mathrm{~cm})$ was achieved at $\mathrm{T}_{3}(115,40,110$ and $25 \mathrm{~kg}$ $\mathrm{N}, \mathrm{P}, \mathrm{K}$ and $\mathrm{S} \mathrm{ha}^{-1}$ respectively) treatment and the shortest plant $(22.53 \mathrm{~cm})$ grew from $\mathrm{T}_{14}$ treatment (control). Due to the effect of different combinations of fertilizers significant differences were found on leaf number, flowers, fruits, destroyed fruits and fruit weight plant $^{-1}$, fruit length, fruit diameter, individual fruit weight and fruit yield.
From the result of present investigation it was revealed that -

1. The combined fertilization of $115,40,110$ and $25 \mathrm{~kg} \mathrm{ha}^{-1} \mathrm{~N}, \mathrm{P}, \mathrm{K}$ and $\mathrm{S}$ respectively showed the better performance for most of the characters as well as yield of strawberry.

2. N, P, K and S content of strawberry shoot and fruit increased with increasing rate of N,P,K,S fertilizer application in combination treatment.

3. $\mathrm{N}, \mathrm{P}, \mathrm{K}$ and $\mathrm{S}$ content in strawberry fruit was more than shoot at the same fertilizer combination. 


\section{References}

Albregts, E. E. and Chandler, C. K. 1993. Slow release fertilizer rates for strawberry fruit production. Soc. Hort. Sci., 106:187-189.

BARC (Bangladesh Agricultural Research Council). 2005. Fertilizer recommendation guide, Pub. no. 45. Bangladesh Agricultural Research Council, Farmgate, Dhaka.

Chandal, R. S.; Kalyan, S.; Singh, A. K. and Sudhaker, P.C. 2003. Effect of sulphur nutrition in rice and mustard grown in sequence. Indian J. Plant Physiol., 8(2):155-159.

Darnell, R. L.; Cantliffe, D. J.; Kirschbaum, D. S. and Chandlar, C. K. 2003. The physiology of flowering in strawberry. Hort. Reviews, 28(6):325-332.

Prabhu, M.; Natrarajan, S.; Srinivsan, K. and Pugalendhi, L. 2006. Integrated nutrient management in cucumber. Indian J. Agric. Res., 40(2):23-126.

Spayd, S. E. and Morris, J. R. 1981. Physical and chemical characteristics of puree form once-over harvest strawberries. J. A. Hort. Sci., 106:101-105.

Wills, R.; Mc Glasson, B.; Graham, D. and Joyee, D. 1998. Post-harvest: an introduction to the physiology and handling of fruit, vegetables and ornamental. UNSW Press, Adelaide, South Australia. $262 \mathrm{p}$. 